\title{
LA A23 SI RACCONTA: UNA VOCE PER CHI INSEGNA NELLA CLASSE DI CONCORSO DI LINGUA ITALIANA PER DISCENTI DI LINGUA STRANIERA
}

\author{
Igor Deiana, Stefania Malavolta, Carla Marulo ${ }^{1}$
}

\section{INTRODUZIONE}

Sono numerosi i lavori che nel corso degli ultimi decenni hanno messo in luce come la scuola italiana si caratterizzi per l'assenza di docenti esperti e competenti nell'ambito della didattica dell'italiano $\mathrm{L}^{2}$. $\mathrm{E}$ innegabile come questa debolezza, alla quale si aggiungono la generale mancanza di un'adeguata formazione iniziale del corpo docente e l'insufficienza dei piani per la formazione in servizio, abbia avuto forti ricadute negative sulla qualità degli interventi di integrazione linguistica rivolti alle studentesse e agli studenti alloglotti. Nonostante in Italia non mancassero e non manchino docenti di italiano L2 ${ }^{3}$, fino al 2016 l'assenza di una classe di concorso dedicata a questa disciplina ha impedito a questi professionisti di lavorare con regolarità nella scuola. Ė per questo motivo che, nel febbraio 2016 l'istituzione della classe di concorso di Lingua italiana per discenti di lingua straniera, la cosiddetta A23, fu accolta positivamente in quanto sembrava colmare questo vuoto del sistema scolastico.

Malgrado la creazione della classe di concorso A23 abbia rappresentato un riconoscimento istituzionale per l'italiano L2 nella scuola, in accordo con quanto emerso da APIDIS (2019), Igor Deiana (2020, 2021) e Igor Deiana e Stefania Spina (2020), sembra sia cambiato ben poco rispetto a quanto atteso. Infatti, pur essendo gli unici insegnanti della scuola italiana che sulla base di titoli ed esperienza possono essere considerati competenti in didattica dell'italiano L2, le e i docenti A23 costituiscono una piccola minoranza del corpo docente in servizio, assegnata esclusivamente ai Centri Provinciali di Istruqione per Adulti (d'ora in avanti CPIA) ${ }^{4}$.

Visto che i CPIA sono l'unica scuola in cui al momento le e i docenti A23 lavorano, l'evento A023: un confronto di esperienze organizzato in occasione di FIERIDA 2021 è stato

\footnotetext{
${ }^{1}$ Igor Deiana (Università per Stranieri di Perugia), Stefania Malavolta (CPIA metropolitano di Bologna), Carla Marulo (CPIA metropolitano di Bologna).

2 Si rimanda a Ciliberti (2008), Diadori (2010), Lo Duca (2013), Grosso (2013), Giardini (2016), Scaglione (2017), Andreoletti (2018), Troncarelli, La Grassa (2018) e Deiana, Spina (2020).

3 È ormai pluridecennale l'offerta formativa per insegnanti di italiano L2 (corsi di laurea triennale, specialistica e magistrale, master di I e II livello, certificazioni di competenza, corsi di perfezionamento e scuole di specializzazione) offerta da diverse università italiane.

${ }^{4}$ I CPIA costituiscono una tipologia di istituzione scolastica autonoma dotata di un proprio organico e di uno specifico assetto organizzativo e didattico secondo quanto stabilito dal Decreto del presidente della Repubblica n. 263 del 29 ottobre 2012 e dalle Linee guida per il passaggio al nuovo ordinamento a sostegno dell'autonomia organizzativa e didattica dei Centri provinciali per l'istruąione degli adulti pubblicate con il decreto ministeriale n. 26 del 12 marzo 2015 (Colosio, 2015). I CPIA organizzano percorsi di istruzione finalizzati al conseguimento di titoli di studio e certificazioni: i Percorsi di alfabetizzazione e apprendimento della lingua italiana (d'ora in avanti Percorsi AALI), i Percorsi di primo livello e i Percorsi di secondo livello; iniziative di ampliamento dell'offerta formativa (prevalentemente corsi di italiano L2, corsi di lingua straniera e corsi di informatica); e attività di ricerca in materia di istruzione degli adulti attraverso i Centri Regionali di Ricerca, Sperimentazione e Sviluppo noti anche come CRRS\&S (Pitzalis, 2019).
} 
un'importante occasione per sondare l'attuale situazione di questa classe di concorso. L'iniziativa, inoltre, oltre a evidenziare problemi e criticità ${ }^{5}$, ha permesso la condivisione di buone pratiche e la proposta di azioni costruttive.

Dopo una breve presentazione di FIERIDA e di A023: un confronto di esperienze, il contributo si propone di fare un punto sul ruolo che le e i docenti A23 hanno nei CPIA attraverso le dichiarazioni rilasciate nel corso dell'iniziativa. Inoltre, sempre grazie alle osservazioni fatte dalla platea partecipante, sono presentate una serie di proposte attuabili e concrete per un migliore impiego della professionalità di questi docenti nei CPIA e nelle scuole secondarie di primo e secondo grado.

\section{FIERIDA}

FIERIDA è un evento di tre giornate organizzato ogni anno dalla Rete Italiana Istruzione degli Adulti (d'ora in avanti RIDAP) ${ }^{6}$ che attraverso sessioni plenarie e momenti paralleli di approfondimento e dibattito cerca di favorire la riflessione sul sistema dell'Istruzione degli Adulti italiano. L'evento si propone di dar vita a un confronto tra i CPIA e il mondo della formazione, dell'università, della ricerca, dell'editoria e del lavoro. Dopo la prima edizione tenutasi a Napoli nel $2017^{7}$, ogni anno l'evento è stato dedicato a uno specifico aspetto dell'istruzione degli adulti. Nel 2018 FIERIDA si è svolta a Torino ${ }^{8}$, nel 2019 a Siena ${ }^{9}$ e nel 2021, a causa delle restrizioni dovute alla pandemia di Covid19, si è tenuta in modalità online ${ }^{10}$.

In occasione di quest'ultima edizione il comitato organizzativo, che aveva come istituto capofila il CPIA metropolitano di Bologna, ha dedicato una delle 56 sessioni parallele alla classe di concorso A23. I contenuti e gli obiettivi di questo webinar, intitolato A023: un confronto di esperienze, sono descritti di seguito nel paragrafo 3.

\section{A023: UN CONFRONTO DI ESPERIENZE}

Tenutosi il 15 maggio 2021 dalle 9:00 alle 11:00, il webinar A023: un confronto di esperienze si proponeva di favorire la messa in comune di buone pratiche in un'ottica costruttiva e di facilitare la creazione di rete di docenti A23.

\footnotetext{
${ }^{5}$ Si ricorda come la classe di concorso A23 sin da prima della sua creazione sia stata al centro di numerose polemiche e discussioni. Per ripercorrerne in modo più approfondito e dettagliato la storia e i problemi si rimanda a Giardini (2016), APIDIS (2019), Deiana (2020) e Deiana, Spina (2020).

${ }^{6} \mathrm{La}$ RIDAP è una rete che si propone di costituire un sistema che faciliti i rapporti tra le principali istituzioni che si occupano di educazione degli adulti e l'amministrazione centrale, gli enti pubblici e privati, le associazioni di categoria e le organizzazioni professionali e sindacali. Possono aderirvi i CPIA e le scuole secondarie di secondo grado in cui sono istituiti i percorsi di secondo livello.

7 Intitolata La scuola degli Adulti, la prima edizione di FIERIDA ha avuto luogo nella Città delle Scienze di Napoli tra il 29 e il 31 maggio 2017. A conclusione dell'iniziativa sono stati contati: 1.600 visitatori provenienti da 17 regioni italiane durante le tre giornate (media di oltre 500 visitatori al giorno), 23 relatori istituzionali, 40 CPIA partecipanti come relatori, 17 stand di reti regionali di CPIA, 10 stand di aziende, 4 sessioni istituzionali, 24 spazi per workshop e comunicazioni curati direttamente dai CPIA, 3 sessioni dedicate ai progetti Erasmus+ e 4 laboratori formativi.

${ }^{8}$ Intitolata Adulti al centro dell'istruzione, la seconda edizione di FIERIDA si è tenuta dal 24 al 26 maggio 2018 presso il Campus Luigi Enaudi dell’Università di Torino.

${ }^{9}$ La terza edizione di FIERIDA si è tenuta a Siena presso l'Università per Stranieri dal 25 al 27 settembre 2019, il tema di quest'edizione era L'Istruqione degli Adulti in Italia: Passato, Presente, Futuro.

${ }^{10} \mathrm{La}$ quarta edizione di FIERIDA è stata dedicata a L'Istruz̧ione degli Adulti in Italia oltre il COVID e si è tenuta online dal 13 al 15 maggio 2021: https://www.youtube.com/watch?v=TVLe-Rn7Z14.
} 
Nonostante siano state confermate molte delle criticità già note e siano emerse anche nuove questioni problematiche, come si vedrà nel corso dei prossimi paragrafi, l'incontro ha cercato di mantenere un atteggiamento costruttivo che permettesse di trasformare malcontenti e dissapori in proposte fattive, attuabili e concrete.

Dal momento che la situazione della A23, e ancora più in generale quello degli stessi $\mathrm{CPIA}^{11}$, si caratterizza per una marcata eterogeneità, il webinar, creando una rete tra coloro che hanno lavorato e lavorano in questa realtà, ha messo in comune esperienze e ha offerto un'opportunità per descrivere i contesti che le e i docenti A23 sperimentano nei CPIA delle diverse regioni d'Italia.

\subsection{Chi ha partecipato?}

Il webinar era rivolto prioritariamente a insegnanti della classe di concorso A23 ed era aperto anche a dirigenti scolastici.

Vista la presenza di una fase laboratoriale condotta attraverso un dibattito guidato, inizialmente l'incontro prevedeva un numero massimo di 60 partecipanti così da garantire il confronto e la condivisione di esperienze e idee. Diversamente da quanto previsto, l'interesse si è rivelato molto più ampio e malgrado non tutti gli iscritti abbiano partecipato all'evento, le iscrizioni ricevute tramite l'apposito modulo Google sono state 107.

\section{Grafico 1. Composizione della platea partecipante all'evento ${ }^{12}$}

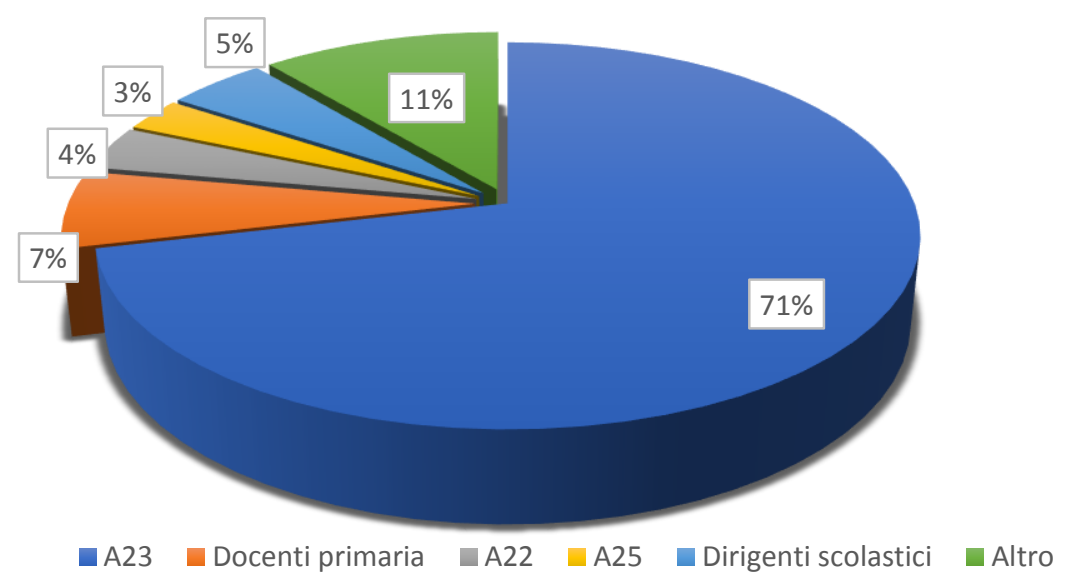

Il Grafico 1 permette di capire chi si sia iscritto al webinar. Più del $70 \%$ delle persone iscritte lavora o ha lavorato come docente A23; la restante parte, oltre alle e ai dirigenti scolastici, era costituita da insegnanti che provenivano da altre classi di concorso: docenti di italiano, storia, geografia, docenti di lingua inglese, docenti della scuola primaria. Questo dato conferma come il campo d'azione e le questioni che coinvolgono la A23 siano trasversali.

${ }^{11} \mathrm{E}$ bene tenere presente come le differenze tra contesti e territori determinino delle differenze, in alcuni casi molto marcate, rispetto alle caratteristiche dell'utenza, del corpo docente e dell'offerta formativa dei vari CPIA italiani.

${ }^{12}$ Di seguito si sciolgono i codici impiegati per indicare le classi di concorso: A22 (Italiano, storia, geografia, nella scuola secondaria di I grado), A25 (Lingua inglese e seconda lingua comunitaria nella scuola secondaria di primo grado). 


\subsection{Modalità di svolgimento dellincontro}

Il webinar è stato organizzato in tre momenti, di cui uno "laboratoriale" finalizzato alla condivisione di esperienze e idee.

Inizialmente ci si è concentrati su alcuni aspetti della storia della A23 utili per inquadrare gli aspetti normativi fondamentali che hanno determinato l'evolversi della classe di concorso.

Dopo questo primo momento in plenaria, le e i partecipanti sono stati divisi in due sottogruppi in cui la discussione si è sviluppata attraverso un dibattito guidato. Al fine di facilitare la condivisione e il confronto, questa sessione interattiva e laboratoriale è stata dedicata esclusivamente a docenti A23 e dirigenti scolastici. In una prima fase, grazie alla lavagna interattiva della funzione Jamboard di Google, è stato chiesto di "postare" il proprio punto di vista su alcune domande-stimolo che si proponevano di raccogliere punti di forza, criticità ed eventuali proposte. Di seguito le tre domande:

1. Rispetto a quello che fai, quali sono i punti di forza dei compiti che ti sono stati affidati in qualità di A023?

2. Nel tuo CPIA il tuo lavoro come A23 potrebbe essere valorizzato meglio? Se sì, come?

3. La normativa prevede due docenti per ogni CPIA; se ce ne fossero di più, che tipo di supporto potrebbero dare? Quali altri incarichi sarebbero utili nel tuo contesto?

In un secondo momento, nel tentativo di raccogliere le buone pratiche e quanto di positivo emerso, gli aspetti più salienti di ciascuna domanda sono stati discussi nei due sottogruppi.

In conclusione, il terzo ed ultimo momento si è svolto nuovamente in sessione plenaria e ha offerto uno spazio di ulteriore discussione e condivisione delle considerazioni espresse nei due sottogruppi.

\section{COSA FA LA A23 NEI CPIA?}

Le informazioni raccolte attraverso il modulo Google compilato per iscriversi al webinar permettono di capire secondo quali modalità le e gli A23 siano impiegati nei CPIA. In continuità con quanto già emerso in Igor Deiana e Stefania Spina (2020) e Igor Deiana (2020, 2021), si conferma come gli A23 svolgano diversi compiti. Se i percorsi AALI sono quelli in cui sono coinvolti maggiormente, il Grafico 2 mette in luce come in generale offrano il loro contributo in diverse attività organizzate dal CPIA.

È bene però evidenziare come le caratteristiche dei contesti, dei territori e delle realtà in cui i vari CPIA sono inseriti determinino delle forti differenze rispetto alle singole esperienze. Anticipando quanto in parte messo in luce durante il dibattito guidato, infatti, sembra che l'assenza di una normativa chiara e di un modus operandi omogeneo abbia avuto conseguenze negative e, conseguentemente, determinato una pluralità di esperienze tra loro molto diversificate. 
Grafico 2. Percorsi di istruzione e attività di ampliamento dell'offerta formativa in cui lavorano le e i docenti A23

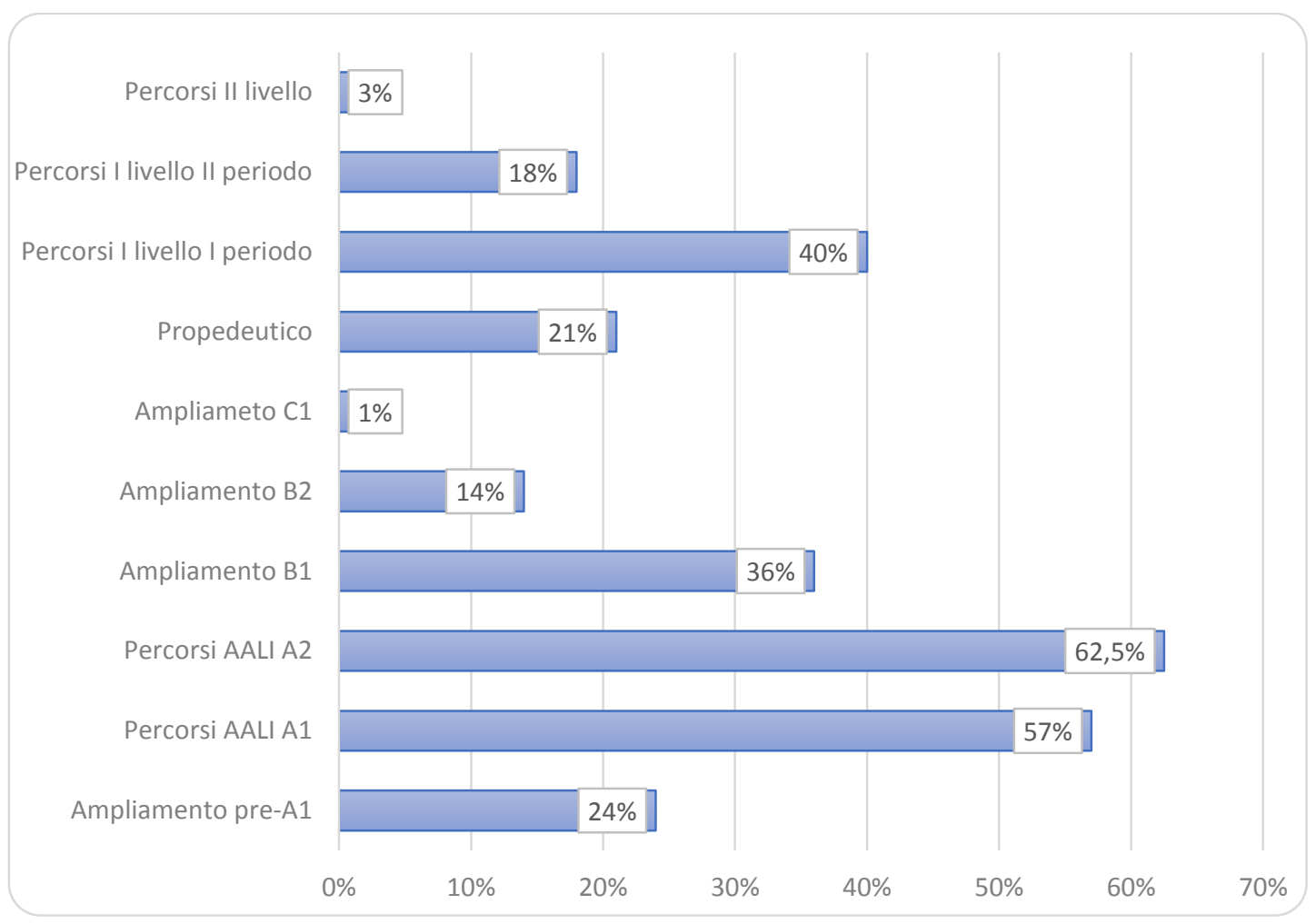

Le e gli A23 sono costretti a fare affidamento sul buon senso della dirigenza scolastica in cui prestano servizio in un contesto in cui le e i dirigenti scolastici sono liberi di gestire le risorse della A23 con eccessiva autonomia. Ancora una volta è emerso un discreto numero di occasioni in cui gli incarichi affidati non erano in linea con quanto si presume dovrebbe essere di competenza di un docente italiano L2. Alcuni docenti sono stati impiegati per insegnare la seconda disciplina in cui sono abilitati, altri per sostituire i colleghi di materia affine assente, come è accaduto a chi ha sostituito i docenti A22 (Italiano, storia, geografia nella scuola secondaria di I grado) o A25 (Lingua inglese e seconda lingua comunitaria nella scuola secondaria di primo grado) nei percorsi di primo livello o a coloro che, utilizzando le parole di uno dei partecipanti all'evento, "fanno i "tappabuchi" dove c’è bisogno (alfabetizzazione, scuola carceraria o percorsi primo livello)».

\section{PUNTI DI FORZA E CRITICITÀ}

L'aspetto positivo unanimemente riconosciuto dalle e dai partecipanti è il traguardo raggiunto grazie alla creazione di questa classe di concorso: per la prima volta si è preso atto dei bisogni formativi in italiano L2 ed è stata riconosciuta una nuova professionalità, prima "in ombra". In diversi interventi è stato sottolineato come la A23 abbia permesso il riconoscimento di una figura professionale che fino a quel momento non faceva parte del corpo docente e a cui gli istituti scolastici ricorrevano solo grazie ad esperti del terzo settore, i cosiddetti "facilitatori linguistici" previsti dalla stessa normativa ministeriale. Come già anticipato, uno degli aspetti che colpisce maggiormente è il forte impatto che l'assenza di una normativa chiara e un di modus operandi condiviso ha avuto sulla pluralità 
delle esperienze registrate. A causa della disomogeneità caratterizzante i CPIA, questa grande flessibilità ha lati positivi ma anche forti criticità e al suo interno ogni singola dirigenza scolastica identifica i propri spazi di autonomia di gestione. Di seguito tre interventi in cui questo aspetto è emerso in modo evidente:

Non è chiaro né per il preside né per i colleghi di ruolo quale sia il compito della figura di A023 all'interno dei CPIA, motivo per cui ogni anno il ruolo cambia, facendo da "tappabuchi" dove c'è bisogno (alfabetizzazione, scuola carceraria, percorsi primo livello).

Il DS dovrebbe tenere presente le specifiche competenze e stabilire come utilizzare il docente (ampliamento offerta formativa? Alfabetizzazione A1A2? L2 sulla LM?) per evitare di creare confusione e malcontento e non valorizzare risorse preziose.

Sarebbe davvero necessario stilare delle linee guida su cosa la A23 può fare (corsi AALI, italiano L2 per lo studio nei percorsi LM, 2 PERIODO...) e cosa no (supplente "tappabuchi", insegnante di inglese/francese, compresenza su inglese/francese...) da diffondere tra DS dei CPIA di tutta Italia. Da lì partire per iniziare ad applicare buone pratiche anche in mancanza di una normativa chiara. Stilare poi un documento più tecnico e farlo arrivare al Ministero e agire sulla normativa.

A causa dell'eterogeneità delle esperienze raccontate dalle e dai partecipanti non è stato possibile stabilire una netta divisione tra punti di forza e criticità. In diverse occasioni aspetti presentati da alcuni come funzionanti e positivi nella loro realtà si sono rivelati essere le più grandi criticità di altri contesti. Riportati di seguito, durante i due laboratori di discussione guidata sono emersi diversi esempi al riguardo:

Posso decidere autonomamente come organizzare le mie attività didattiche con le studentesse/i (impostazione delle UdA, materiali, tempi).

Flessibilità a strutturare la didattica in CAD e quindi con livelli non omogenei. Utilizzo degli strumenti digitali e informatici sempre, anche fuori dal contesto pandemico.

Ci occupiamo sia di italiano L2 nei corsi AALI sia di SEMPLIFICAZIONE dei materiali (materie scientifiche, ecc.).

Faccio quello per cui sono stata assunta e responsabile dell'AALI.

Affiancamento sul $2^{\circ}$ periodo didattico.

Faccio italiano nei corsi di $3^{\wedge}$ media.

Riconosco l'autonomia che mi viene concessa. Essa si riferisce sia al fatto che possa gestire in autonomia l'ora di lezione (non essendo necessariamente in compresenza) sia nel lavorare sul potenziamento di ciò che ritengo opportuno potenziare.

Possibilità di: insegnamento su più livelli, collaborazione con enti esterni, affidamento di incarichi istituzionali. 
Dalle otto testimonianze presentate è possibile rilevare come la creazione dell'A23 abbia reso possibile molteplici interventi. Quanto registrato conferma come le competenze di docenti specializzati e formati in didattica dell'italiano L2 possano essere impiegate in modi differenti nelle attività organizzate dal CPIA. Paradossalmente, è stato possibile vedere come, a causa dell'eterogeneità legata a variabili di contesto e territorio, il potenziale di questi docenti non sia sfruttato al meglio. Infatti, molte testimonianze hanno evidenziato punti critici che richiedono:

maggiore coinvolgimento in corsi alfa e offerta formativa;

più incarichi di responsabilità, anche in scrutinio LM, CLIL, funzione strumentale, possiamo dare prospettive nuove (in un mestiere nuovo);

o che segnalano:

assenza di collaborazione con i docenti dei corsi di alfabetizzazione. Inoltre, tante ore di compresenza non valorizzate.

[che] la professionalità dell'A023 attualmente da noi non è considerata come figura trasversale e facilitatore linguistico per i percorsi di I e II livello.

[come la A23 sia una] figura non riconosciuta dai colleghi.

[come] quest'anno mi è stato affidato un corso di ampliamento in cui lavoro sull'italiano L2 in generale e per le discipline. La principale criticità nella mia esperienza è non poter svolgere se non in piccolissima parte il mio lavoro nella mia disciplina.

[come] l'autonomia e libertà a me concessa diventa un punto di debolezza lì dove non viene concepita la compresenza con la docente di lingua italiana, a mio avviso fondamentale almeno qualche ora per i corsi di primo livello.

Colpisce in particolare la testimonianza di un docente che racconta come:

Dopo due anni di A023 al CPIA, ho rinunciato al ruolo e ho preso un nuovo ruolo su inglese $\mathrm{AB} 25$, poiché la mia figura all'interno del CPIA non riusciva a trovare una collocazione sensata, nonostante le numerose proposte portate. Paradossalmente, ora che sono l'insegnante di potenziato di una Scuola secondaria di primo grado, grazie al mio profilo (specialista italiano L2, specialista Sostegno, poliglotta) lavoro come A023, estendendo il mio campo di azione anche sulla scuola primaria. Come A023 insegnavo inglese, come AB25 insegno italiano L2.

In generale quanto messo in luce nei due sottogruppi può essere riassunto in tre punti. Mentre i primi due confermano come la formazione specifica e l'esperienza maturata nella didattica dell'italiano L2 rappresentino due elementi in cui le e i docenti A23 si riconoscono, il terzo e ultimo si concentra sul loro ruolo nei CPIA.

1. La specifica formazione linguistica e glottodidattica è un punto di forza della A23. Le conoscenze teoriche e la familiarità con metodologie, tecniche e strategie didattiche si rivelano utili e proficue, ad esempio, per identificare i diversi livelli di apprendimento, $\mathrm{i}$ profili delle competenze in entrata e la valutazione in uscita, nei percorsi AALI e più in generale in tutti i corsi di italiano L2, anche quelli erogati nell'ambito dell'ampliamento dell'offerta formativa. La specifica formazione didattica delle e degli A23 favorisce anche 
lo sviluppo di interventi che richiedono specifici percorsi di analisi e riflessione sull'intercultura, sulla dialettica tra identità e diversità, sul rapporto con la diversità culturale nella dimensione dell'incontro, nella condivisione di spazi, nei luoghi istituzionali.

2. Quanto appreso dalle pregresse esperienze di insegnamento in italiano L2 rappresenta, inoltre, un altro punto di forza. Infatti, l'aver già lavorato con apprendenti di italiano L2 portatori di lingue e culture diverse facilita l'inserimento in una realtà come quella del CPIA, in cui ai diversi profili socioculturali sono associati particolari e specifici percorsi di migrazione in età adulta (Troncarelli, La Grassa, 2018).

3. Infine, rispetto al loro ruolo nei CPIA, pur riconoscendo la loro importanza e centralità nei Percorsi AALI, le e gli A23 segnalano l'esigenza di poter lavorare anche nel primo e secondo periodo dei percorsi di primo livello ${ }^{13}$ sui linguaggi disciplinari e/o in laboratori di potenziamento sulla lingua per lo studio. Il ricorso e l'impiego di metodologie e tecniche didattiche per facilitare l'accessibilità ai testi di studio sembra essere una delle principali attività in cui le e gli A23 hanno contribuito nel corso di questi anni di servizio. Oltre a offrire un importante contributo al percorso formativo di apprendenti stranieri, sembra che in alcuni casi le conoscenze e le consapevolezze maturate rispetto ai tempi e alle sequenze dell'apprendimento siano state condivise anche con le e i colleghi meno esperti.

\section{AlCUNE PROPOSTE}

Tenendo presenti le buone pratiche osservate e nel tentativo di reinterpretare le criticità evidenziate in ottica costruttiva, nel corso della terza e ultima parte dell'incontro quanto emerso dai due paralleli laboratori di discussione guidata ha incoraggiato la formulazione di alcune proposte per il futuro.

Argomentate attraverso quanto dichiarato dalla stessa platea partecipante, di seguito si illustrano le sei azioni per un migliore impiego della classe di concorso A23 e un più generale miglioramento della didattica dell'italiano L2 nei CPIA e nella scuola italiana.

1. La prima proposta prevede che, attraverso indicazioni ministeriali, siano stabiliti i compiti dei docenti A23 in modo da riconoscere e valorizzare l'importanza della loro professionalità. Di fatto essi rappresentano già un "collante" e un elemento di continuità fra i percorsi AALI e i percorsi di primo livello, ma potrebbero anche essere coinvolti formalmente in tutte le attività riguardanti l'italiano L2: sessioni di educazione civica, esami della prefettura e attività dei Centri Regionali di Ricerca, Sperimentazione e Sviluppo - CRRS\&S:

Bisognerebbe riconoscere l'importanza dell'A023 come esperto di L2, "pietra angolare" dei percorsi di apprendimento e insegnamento (sul piano della programmazione, progettazione, attività didattica, ricerca e sperimentazione) nei CPIA, a seguito di un ristrutturazione/rimodulazione dell'organico dell'autonomia.

\footnotetext{
13 I percorsi di primo livello si distinguono dai corsi erogati nella scuola del mattino perché «non si suddividono in anni scolastici, ma in periodi didattici, che possono essere affrontati e conclusi dallo studente in tempi diversi a seconda del suo piano di studi personalizzato» (Colosio, 2015: 41). Sono suddivisi in due periodi didattici: il primo periodo didattico permette di conseguire il titolo di studio conclusivo del primo ciclo d'istruzione (corrispondente all'ex licenza media) e il secondo permette il rilascio della certificazione attestante l'acquisizione delle competenze di base connesse all'obbligo di istruzione relative alle attività e insegnamenti generali comuni a tutti gli indirizzi degli istituti professionali e degli istituti tecnici corrispondente al primo biennio della scuola secondaria di secondo grado.
} 
2. La seconda azione prevede l'incremento del numero di cattedre assegnate a ruolo poiché le due cattedre assegnate sono insufficienti. Infatti,

portare più A23 nei CPIA garantirebbe personale con formazione specifica e con una certa "visione" della scuola, che potrebbe contribuire a far crescere l'Istruzione per adulti.

Inoltre,

Un maggior numero di A023 consentirebbe un impiego ottimale delle CDC in tutte le sedi in percorsi di 200 ore in affiancamento ai docenti di disciplina, nel primo e secondo periodo in compresenza con i docenti di disciplina per un POTENZIAMENTO delle competenze linguistiche nei linguaggi specifici delle discipline e nell'organizzazione di corsi di ampliamento di italiano L2 nei livelli oltre l'A2, in corsi di preparazione alle certificazioni linguistiche, nella formazione interna dei docenti di altra materia sulle specificità dell'insegnamento dell'italiano L2 e lingua di studio.

Quanto detto risulta ancora più evidente nella testimonianza che racconta come

Nella mia realtà sono presenti i posti di L2 ma non 2 nello stesso plesso quindi aumentare $\mathrm{i}$ posti potrebbe essere utile in primis per poter collaborare in sinergia all'interno delle stesse classi. Ciò permetterebbe una maggiore progettualità e magari anche una sorta di ricerca sul campo. Di conseguenza diverse figure di ItL2 sarebbero una risorsa all'interno delle diverse classi agendo in qualità di supporto sia in italiano che nell'apprendimento delle diverse discipline.

Rispetto a questo secondo punto, quanto dichiarato da una dirigente scolastica sembra offrire una valida soluzione. Infatti, si propone che al momento di un pensionamento $i$ CPIA richiedano un numero maggiore di "cattedre a ruolo" per la A23. La dirigente ha raccontato come

Nel mio CPIA non sono mai stati attribuiti i posti (almeno 2) di A23 sull'organico potenziato. Per il prossimo a.s. (2021/2022) ci sono stati attribuiti finalmente 3 posti in organico potenziato a seguito del pensionamento di 3 unità di scuola primaria. Nel corso degli anni è stato possibile incrementare il numero di A23 in organico di diritto a seguito del pensionamento di alcuni docenti su classi di concorso differenti, richiedendo il cambio di classe di concorso senza creare soprannumerari.

3. La terza proposta prevede che chi insegna nei corsi AALI abbia almeno una certificazione in didattica dell'italiano L $2^{14}$. Infatti,

Bisognerebbe richiedere a chi è di ruolo quantomeno il possesso di una certificazione di didattica dell'italiano a stranieri (Cedils, Ditals,...). E poi iniziare a colmare i posti vacanti con le A23 e non attingere dalle graduatorie della primaria.

$\mathrm{Al}$ momento mi occupo dei corsi AALI pur avendo una lunga esperienza come facilitatrice nelle scuole primarie e secondarie di primo grado: potrei

14 È bene ricordare come questi titoli abbiano come requisito essenziale, fra gli altri, un numero considerevole di ore di esperienza pratica di insegnamento della lingua seconda. 
offrire supporto ai colleghi della licenza media. Rilevo che troppo spesso al CPIA arrivano docenti senza preparazione didattica per l'insegnamento della lingua 2: importante considerare di più il curriculum e le competenze specifiche dei docenti.

Si specifica come questa richiesta non debba essere letta come un atto di prevaricazione nei confronti delle e dei colleghi della scuola primaria. Infatti, in diverse occasioni è emerso come «il lavoro del maestro e del docente di A023 è complementare e funziona perfettamente se è svolto in sinergia». Questa richiesta, infatti, non ambisce all'esclusione dei docenti già in servizio ma, parallelamente a quanto richiesto per la $\mathrm{A} 23$, prevede che coloro che si occupano di italiano L2 siano in possesso di titoli per cui possono essere ritenuti competenti nell'ambito della didattica dell'italiano L2.

4. La quarta proposta mira a valorizzare le ore di compresenza nei percorsi di primo livello. In questo modo sarebbe possibile lavorare sulle competenze e le abilità dell'ItalStudio ${ }^{15}$ e facilitare gli apprendimenti disciplinari. Inoltre, sarebbe bene inserire le e gli A23 anche nei percorsi di 200 ore propedeutici ai percorsi di primo livello: infatti, sarebbe così possibile inserire specifiche attività rivolte alle e agli alloglotti anche nella fase che precede e accompagna al conseguimento dell'ex Licenza Media.

Il docente itL2 dovrebbe essere sia docente di lingua italiana sia supporto linguistico durante l'apprendimento disciplinare, in compresenza con i docenti delle differenti discipline.

Dovremmo avere la possibilità di dedicarci anche all'italiano per lo studio perché i ragazzi che hanno conseguito A2 ed entrano nei percorsi di primo periodo si trovano completamente spaesati di fronte ai testi delle materie scientifiche; quindi, semplificazione dei testi scienze, tecnologia e matematica.

5. La quinta proposta prevede che alla A23 siano affidati momenti di supporto rivolti alle e ai colleghi con minori competenze e formazione rispetto all'italiano L2 e l'affiancamento ai neoassunti privi di qualsiasi nozione in didattica dell'italiano L2. La diffusione di buone pratiche e di valide metodologie glottodidattiche affidata alla A23 potrebbe avere delle ricadute positive sulla qualità della didattica dell'italiano L2 all'interno di ogni CPIA. Secondo quanto emerso dalle diverse esperienze raccolte, le e gli A23 potrebbero

elaborare test di ingresso per i corsi di alfabetizzazione e apprendimento della lingua italiana e per i corsi di primo livello primo periodo (per indirizzare gli studenti con livello inferiore all'A2 ai corsi di alfabetizzazione); tenere corsi di formazione per i colleghi (ad esempio su italiano lingua dello studio, approccio umanistico-affettivo, uso di video nella didattica ecc.).

\section{Per far questo sarebbe necessaria}

Maggiore collaborazione con i colleghi di primo livello e [che la A23 sia riconosciuta] come figura specializzata ed esperta della materia di raccordo tra docenti di Italbase e Italstudio.

\footnotetext{
${ }^{15}$ Nei documenti ministeriali con l'etichetta ItalStudio si fa riferimento alla padronanza della lingua per lo studio, meglio nota nella letteratura internazionale come Cognitive Academic Language Proficiency (Cummins, 1979). Nei documenti ministeriali questa definizione è solitamente affiancata a quella di ItalBase, la quale si riferisce alle necessità di primo livello ossia, sulla padronanza strumentale della lingua di base e nota anche come Basic Interpersonal Communication Skills (Cummins, 1979).
} 
Malgrado non direttamente legata all'esperienza nei CPIA, la sesta proposta è quella che più di tutte sembra aver unito le e i partecipanti all'evento; infatti, richiede l'inserimento della A23 nell'organico della scuola secondaria di primo e di secondo grado. Questa richiesta offre uno sguardo che va oltre l'esperienza dei CPIA: infatti, è importante ribadire come la formazione e l'esperienza di docenti competenti nell'ambito della didattica dell'italiano L2 possa rappresentare un valido aiuto per l'integrazione scolastica degli studenti con background migratorio che frequentano la "scuola del mattino". Avvalendosi delle competenze di questi professionisti, si potrebbero attenuare se non addirittura annullare le disparità e le differenze che intercorrono tra loro e gli studenti italofoni. Infatti,

Se le/i docenti di A023 fossero di più e fossero a sistema non solo nei Cpia ma anche nelle scuole di ogni ordine e grado, probabilmente interventi di qualità di facilitazione linguistica e potenziamento ItalBase e ItalStudio diventerebbero la norma. Il ruolo dei/delle Dirigenti può essere fondamentale per valorizzare docenti con specifica formazione ed esperienza e forse dovrebbero essere anche loro, in prima linea, a segnalare a chi di competenza le criticità che emergono nell'assenza di organico specializzato.

\section{CONCLUSIONI}

Quanto emerso dalle testimonianze delle e dei docenti che hanno partecipato al webinar A023: un confronto di esperienze evidenzia come dopo ormai quasi sei anni dalla creazione della classe di concorso di Lingua italiana per discenti di lingua straniera la formazione e le competenze dei docenti A23 non siano pienamente sfruttate nei CPIA e siano ancora rimaste escluse dalle cosiddette "scuole del mattino".

Al fine di dare un nuovo slancio all'A23, il webinar si propone di essere il primo di una più ampia serie di occasioni di confronto e di non rimanere un'esperienza isolata e per questo ha cercato di diffondere quanto messo in luce dall'incontro attraverso diversi canali. A conclusione dell'evento, il comitato organizzativo ha inviato un documento di restituzione ai partecipanti e, in un secondo momento, alle direzioni di tutti i CPIA italiani: sulla base di quanto osservato, l'invio alle e ai dirigenti di un documento riguardante le buone pratiche e le possibili attività in cui la A23 può essere coinvolta è stato ritenuto una valida azione atta a un riscatto di questa classe di concorso.

Nella speranza che la situazione descritta in questo contributo possa presto migliorare, si riportano le parole pronunciate dal Ministro Patrizio Bianchi in apertura di FIERIDA (13 maggio 2021) che diventeranno realtà tangibile solo risolvendo definitivamente il vuoto normativo che caratterizza la scuola italiana rispetto all'italiano L2.

Per quanti sono arrivati nel nostro paese, la conquista della lingua è fondamentale perché se tu non hai le parole per dirlo qualcun altro lo dice per te, oppure rischi sempre di rimanere alla porta del paese in cui arrivi. 


\section{RIFERIMENTI BIBLIOGRAFICI}

APIDIS (2019), "La classe di concorso A023: una promessa delusa?”, in LinguaInAqione, 11 , pp. 15-24.

Andreoletti C. (2018), "Il docente A23: formazione e competenze", in Italiano a stranieri. Rivista semestrale per l'insegnamento dell'italiano come lingua straniera/seconda, 23, pp. 15-24.

Ciliberti A. (2008), "L'insegnante di italiano lingua non materna: prospettive formative", in Ciliberti A. (a cura di), Un mondo di italiano, Guerra Edizioni, Perugia, pp. 93-104.

Colosio O. (2015), Il nuovo sistema di istruzione degli adulti. Dai CTP ai CPIA, Loescher, Torino.

Cummins J. (1979), "Cognitive/Academic Language Proficiency, Linguistic Interdependence, the Optimum Age Question and Some Other Matters", in Working Papers on Bilingualism, 19, pp. 197-205.

Deiana I. (2020), "Ultimi aggiornamenti dal pianeta A23", in Scuola e Lingue Moderne SeLM, 7-6/2020, pp. 20-25.

Deiana I. (2021), “A23: facciamo il punto!”, in Lingue e Linguaggi, 41, pp. 101-117: http://siba-ese.unisalento.it/index.php/linguelinguaggi/article/view/23304.

Deiana I., Spina S. (2020), "Breve storia della classe di concorso A23-lingua italiana per discenti di lingua straniera", in Italiano LinguaDue, 12, 2, pp. 1-19: https://riviste.unimi.it/index.php/promoitals/article/view/14969.

Diadori P. (2010), Formazione, qualità e certificazione per la didattica delle lingue moderne in Europa, Le Monnier, Firenze-Milano.

Favaro G. (2016), "L'italiano che include: la lingua per non essere stranieri. attenzioni e proposte per un progetto di formazione linguistica nel tempo della pluralità" in Italiano LinguaDue, 8, 1, pp. 1-12:

https:// riviste.unimi.it/index.php/promoitals/article/view/7560.

Giardini D. (2016), "Una lingua seconda per l'italiano: quali scenari per la disciplina e la professione", in Bollettino Itals, 14, 66, pp. 30-56.

Grosso G. (2013), "La formazione del docente di italiano a immigrati adulti", in Benucci A. (a cura di), Formazione e pratiche didattiche in italiano L2, OL3, Perugia, pp. 35-56.

Lo Duca M. G. (2013), Lingua italiana ed educarione linguistica. Tra storia, ricerca e didattica, Carocci, Roma.

Pitzalis M. (2019), "Una sfida per la scuola. I CPIA come punto di incontro tra policy formative e politiche migratorie in Italia", in Colombo M., Scardigno F. (a cura di), La formazione dei rifugiati e dei minori stranieri non accompagnati. Una realtà necessaria, Vita e Pensiero, Milano, pp. 37-46.

Scaglione S. (2017), "La diversità linguistica a scuola: un mondo (ancora) inesplorato", in Vedovelli M. (a cura di), L'italiano dei nuovi italiani. Atti del XIX Convegno nazionale del GISCEL, Aracne, Roma, pp. 211-226.

Troncarelli D., La Grassa M. (2018), La didattica dell'italiano nel contato interculturale, il Mulino, Bologna. 